\title{
Sobrecarga del cuidador del paciente con tratamiento de diálisis peritoneal
}

\author{
Requilda Angélica Cubas Sánchez, Narda Patricia Sánchez Gamarra, Norma del Carmen Gálvez Díaz \\ Facultad de Ciencias de la Salud. Escuela de Enfermería. Universidad Señor de Sipán. Chiclayo. Perú \\ Como citar este artículo: \\ Cubas Sánchez RA, Sánchez Gamarra NP, Gálvez Díaz NC. Sobrecarga del cuidador del paciente \\ con tratamiento de diálisis peritoneal. Enferm Nefrol. 2019 Jul-Sep;22(3):317-22
}

\section{Resumen}

Introducción: La experiencia de ser cuidador produce sensación de satisfacción por el cuidado y búsqueda de bienestar del paciente, pero desafortunadamente la duración del tiempo, técnica y procedimiento que implica la Diálisis Peritoneal produce sobrecarga en el cuidador, sumado a esto la dependencia del paciente según su edad, estado mental y físico. El objetivo fue determinar el nivel de sobrecarga del cuidador del paciente con tratamiento de Diálisis Peritoneal en una zona aledaña a la ciudad de Chiclayo-Perú.

Material y Método: Estudio cuantitativo, diseño no experimental transversal descriptiva, el muestreo fue aleatorio simple sin reposición, conformado por 75 cuidadores seleccionados por criterios de inclusión y exclusión. La recolección de datos se realizó mediante el cuestionario de sobrecarga de cuidadores de pacientes de Diálisis Peritoneal, previamente validado por tres expertos y con una confiabilidad de 0,847 (alfa de Cronbach). Los datos se procesaron con el software spss versión 22 , en todo momento se respetaron los principios éticos y criterios de rigor científico.

Resultados: $94,7 \%$ de los cuidadores presentaron un nivel de sobrecarga leve, se evaluó tres dimensiones: la dependencia del paciente 53,3\%, auto desempeño del cuidador $96,0 \%$ y las repercusiones socio familiares $78,7 \%$.

Requilda Angélica Cubas Sánchez

Lic. Enfermería de la Universidad Señor de Sipán

Calle Machupicchu, 1105. La Victoria. Chiclayo. Perú

E-mail: angelica_15_2_7@hotmail.com
Conclusión: Podemos concluir que, el nivel de sobrecarga del cuidador es leve, y el perfil más prevalente respecto a los datos sociodemográficos de los cuidadores es mujer adulta, esposa del paciente y que vive con el paciente.

PALABRAS CLAVE: diálisis peritoneal; sobrecarga; cuidador.

\section{Caregiver burden in patients with peritoneal dialysis}

\begin{abstract}
Introduction: The experience of being a caregiver produces a sense of satisfaction for the care and search for patient well-being. Unfortunately, the duration of time, technique and procedure of peritoneal dialysis produces an overload in the caregiver, added the dependence of the patient according to age, mental and physical state. The objective was to determine the level of caregiver burden in patients with peritoneal dialysis treatment in an area adjacent to the city of Chiclayo-Peru.
\end{abstract}

Methodology: Descriptive cross-sectional study. The sampling was simple random without replacement, consisting of 75 caregivers selected by inclusion and exclusion criteria. Data collection was performed using the caregiver burden questionnaire in patients on peritoneal dialysis, previously validated by three experts and with a reliability of 0.847 (Cronbach's alpha). The data were processed with the statistical package SPSS version 22. At all times, ethical principles and criteria of scientific rigor were respected.

Results: $94.7 \%$ of the caregivers presented a level of slight burden, three dimensions were evaluated: the 
dependence of the patient $(53.3 \%)$, self-care of the caregiver (96.0\%) and socio-family repercussions (78, $7 \%)$.

Conclusion: We can conclude that the level of caregiver burden is mild, and the most prevalent profile regarding socio-demographic data of caregivers is: adult female, patient's wife and living with the patient.

Keywords: peritoneal dialysis; burden; caregiver.

\section{Introducción}

La Organización Panamericana de la Salud/Organización Mundial de la Salud (OPS/OMS) y la Sociedad Latinoamericana de Nefrología e Hipertensión (SLANH) llevan tiempo hablando de la necesidad de prevenir y mejorar el acceso a los pacientes con enfermedad renal crónica (ERC), la cual afecta a cerca del $10 \%$ de la población mundial ${ }^{1}$. Según datos de la SLANH en 2011, en América Latina un promedio de 613 pacientes por millón de habitantes tuvo acceso a alguna de las alternativas de tratamiento renal sustitutivo, ya fuera hemodiálisis, diálisis peritoneal o el trasplante de riñón. Sin embargo, la distribución de estos servicios no es equitativa y en algunos países esa cifra fue menor a 200 pacientes por millón de habitantes ${ }^{1}$.

La oferta de servicios de diálisis activos en las diferentes regiones del Perú, evidencian que esta se centra en Lima $(44,8 \%)$ y Callao (12,3\%). Las regiones de Piura, Lambayeque y la Libertad del norte peruano son regiones que ofertan en promedio el $6 \%$ de la oferta total de servicios del país; asimismo, Arequipa ubicada al sur del Perú aporta el $6 \%$ de la oferta total. Llama la atención las tasas menores al $1 \%$ que aportan las regiones de Tumbes, Amazonas, San Martín, Ucayali, Huancavelica, Ayacucho, Apurímac, Madre de Dios y Moquegua y una tasa de 0\% de la Región Pasco².

La diálisis peritoneal domiciliaria es un tratamiento seguro, efectivo y se puede extender a muchos pacientes que hoy no están recibiendo tratamiento. La diálisis peritoneal domiciliaria se ofrece actualmente a alrededor del $12 \%$ de los pacientes en América Latina, la cifra supera el $30 \%$ en algunos países, pero en otros es aproximadamente del $6 \%^{1}$, siendo las poblaciones alejadas de los centros de salud las que más realizan esta terapia. EI soporte de la enfermera en diálisis peritoneal es imprescindible para facilitar la participación del familiar en el tratamiento, desde el aprendizaje, seguimiento y apoyo al cuidador. La edad avanzada y alto nivel de comorbilidad de los pacientes en diálisis peritoneal (DP) ocasiona que necesiten de un cuidador para realizar el tratamiento de manera segura. Por ello, la visión de la enfermera respecto a involucrar en el tratamiento al cuidador es fundamental para brindar cuidados de salud adecuados $^{3}$. Cuando se inicia la indicación de DP, los pacientes y cuidadores se envuelve en un sinfín de interrogantes e incertidumbres respecto al tratamiento. Este cambio en la vida de los cuidadores suele ser afrontado de manera óptima. Sin embargo, hay ocasiones que les genera una desestructuración, que repercute directamente en sus dimensiones físicas, emocionales y psicológicas. Los pacientes con patologías crónicas y con tratamiento prolongado requiere no solo la presencia de un cuidador sino del compromiso que este asuma ciertas partes fundamentales en la vida diaria del paciente.

El Ministerio de salud del Perú no considera dentro de sus políticas estrategias que involucren al cuidador principal de pacientes que reciben DP. Por ello profundizar en el conocimiento de esta problemática permite tener evidencias para proponer políticas de salud que los incluya. Por tanto, el objetivo del estudio es determinar el nivel de sobrecarga del cuidador del paciente en tratamiento con DP en Chiclayo, Perú.

\section{Material y Método}

Se realizó un estudio observacional, transversal y descriptivo. Se incluyeron a todos los cuidadores con más de 6 meses de ayuda continua a pacientes de la unidad de DP entre 18 y 75 años. Las variables analizadas fueron la edad, sexo, parentesco, trabajo, vive con el paciente, estado civil, grado de instrucción, tiempo de ayuda, dependencia del paciente según la percepción del cuidador, auto desempeño del cuidador y repercusiones sociofamiliares. La recogida de datos se realizó mediante un cuestionario para medir la sobrecarga de los cuidadores de pacientes en DP durante el periodo comprendido entre el 20 de marzo y el 31 de mayo de 2017.

El cuestionario de sobrecarga de cuidadores de pacientes de Diálisis Peritoneal evaluó la dependencia, auto desempeño y las repercusiones sociofamiliares según la percepción del cuidador; para cada una de ellas, el cuidador indicó la frecuencia de cómo se siente así mismo y evaluó al paciente, utilizando una escala tipo Likert. Se utilizó la valoración por cuartiles para cada dimensión; en la dependencia del paciente según la per- 
cepción del cuidador (leve: hasta 21 puntos, moderada: de 22 a 25, intensa: más de 26); auto desempeño del cuidador (leve: hasta 29 puntos, moderada: de 30 a 34, intensa: más de 35$)$; repercusiones socio familiares según la percepción del cuidador (leve: hasta 26 puntos, moderada: de 27 a 34, intensa: más de 35), cada dimensión con su respectivo baremo. La puntuación final que representa el grado de sobrecarga del cuidador se obtuvo con el siguiente baremo: leve (hasta 80); moderada (de 81 a 89) e intensa (más de 90). Este cuestionario fue utilizado en España, por Teixidó y Cols ${ }^{4}$, y adaptado para garantizar su interpretabilidad mediante el juicio de tres expertos, la cual estuvo conformada por la enfermera jefa de la Unidad de Diálisis Peritoneal, enfermera jefa del servicio de Nefrología y el médico jefe del Departamento de Nefrología. Los datos se analizaron en el programa estadístico SPSS 22. Se empleó la estadística descriptiva para resumir los resultados en tablas y gráficos. Se solicitó el consentimiento informado a los cuidadores al inicio del estudio.

\section{Resultados}

Se analizaron a 75 cuidadores de pacientes procedentes de la unidad de DP del Hospital de EsSalud de Chiclayo. Se obtuvo el perfil más prevalente respecto a los datos sociodemográficos de los cuidadores, siendo mujer $(74,7 \%)$ adulta $(69,3 \%)$, esposa del paciente $(38,7 \%)$ y que vivían con el paciente $(89,3 \%)$. Conjuntamente, compaginaban el rol de cuidador y el trabajo laboral $(56,0 \%)$ y con un grado de instrucción superior $(54,7 \%)$ con un rango entre $1-3$ años (53,3\%) (Tabla 1$)$.

Se observaron tres niveles fundamentales en cuanto al nivel de sobrecarga del cuidador. Del total de cuidadores, se obtuvo que el $94,7 \%$ presentaban sobrecarga leve, el $2,7 \%$ sobrecarga moderada, y por último el 2,7\% sobrecarga intensa (Figura $\mathbf{1}$ ).

En cuanto a las dimensiones de la sobrecarga del cuidador, la dimensión de dependencia del paciente mostró que el $53,3 \%$ de pacientes tienen dependencia leve, $29,3 \%$ dependencia moderada y $17,3 \%$ dependencia intensa. Según la dimensión del auto desempeño del cuidador, se obtuvo que el $96,0 \%$ presentaron un auto desempeño leve y $4,0 \%$ auto desempeño intensa. Finalmente, la dimensión de repercusiones socio familiares, se demostró que el $78,7 \%$ de los cuidadores presentaban repercusiones leves referente a la vida sociolaboral y tiempo libre, el $14,7 \%$ moderada y el $6,7 \%$ intensa (Figura 2).
Tabla 1. Datos sociodemográficos del cuidador del paciente con tratamiento de Diálisis Peritoneal.

\begin{tabular}{|c|c|c|c|}
\hline Datos & Categoría & $\mathbf{n}$ & $\%$ \\
\hline Edad & $\begin{array}{c}\text { Adolescente } \\
\text { Adulto Joven } \\
\text { Adulto Maduro } \\
\text { Adulto Mayor }\end{array}$ & $\begin{array}{c}4 \\
8 \\
52 \\
11\end{array}$ & $\begin{array}{c}5,3 \% \\
10,7 \% \\
69,3 \% \\
14,7 \%\end{array}$ \\
\hline Sexo & $\begin{array}{l}\text { Femenino } \\
\text { Masculino }\end{array}$ & $\begin{array}{l}56 \\
19\end{array}$ & $\begin{array}{l}74,7 \% \\
25,3 \%\end{array}$ \\
\hline Parentesco & $\begin{array}{c}\text { Hijo/a } \\
\text { Esposo/a } \\
\text { Padre } \\
\text { Madre } \\
\text { Otros }\end{array}$ & $\begin{array}{c}25 \\
29 \\
3 \\
6 \\
12\end{array}$ & $\begin{array}{c}33,3 \% \\
38,7 \% \\
4,0 \% \\
8,0 \% \\
16,0 \%\end{array}$ \\
\hline Trabaja & $\begin{array}{c}\text { Si } \\
\text { No } \\
\text { Estudia }\end{array}$ & $\begin{array}{l}42 \\
22 \\
11\end{array}$ & $\begin{array}{l}56,0 \% \\
29,3 \% \\
14,7 \%\end{array}$ \\
\hline Vive con el Paciente & $\begin{array}{l}\mathrm{Si} \\
\text { No }\end{array}$ & $\begin{array}{c}67 \\
8\end{array}$ & $\begin{array}{l}89,3 \% \\
10,7 \%\end{array}$ \\
\hline Estado Civil & $\begin{array}{c}\text { Soltero/a } \\
\text { Casado/a } \\
\text { Conviviente }\end{array}$ & $\begin{array}{c}27 \\
42 \\
6\end{array}$ & $\begin{array}{c}36,0 \% \\
56,0 \% \\
8,0 \%\end{array}$ \\
\hline Grado de Instrucción & $\begin{array}{l}\text { Primaria } \\
\text { Secundaria } \\
\text { Superior }\end{array}$ & $\begin{array}{c}7 \\
27 \\
41\end{array}$ & $\begin{array}{c}9,3 \% \\
36,0 \% \\
54,7 \%\end{array}$ \\
\hline Tiempo de Ayuda & $\begin{array}{c}1-3 \text { años } \\
4-7 \text { años } \\
8-11 \text { años } \\
12-15 \text { años } \\
16-19 \text { años }\end{array}$ & $\begin{array}{c}40 \\
21 \\
11 \\
2 \\
1\end{array}$ & $\begin{array}{c}53,3 \% \\
28,0 \% \\
14,7 \% \\
2,7 \% \\
1,3 \%\end{array}$ \\
\hline
\end{tabular}

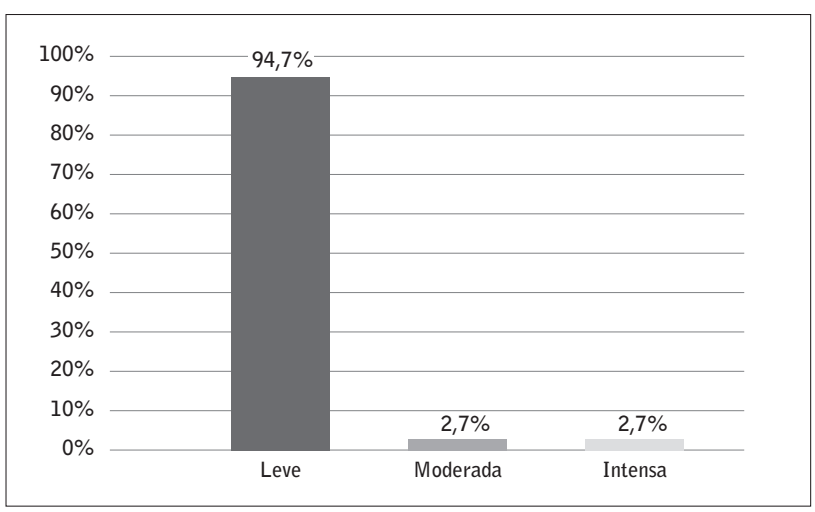

Figura 1. Nivel de sobrecarga del cuidador del paciente con tratamiento de Diálisis Peritoneal.

\section{Discusión}

El estudio ha podido responder al objetivo planteado inicialmente. Prácticamente, podemos observar como la mayoría de la muestra presenta niveles leves en cuanto a 


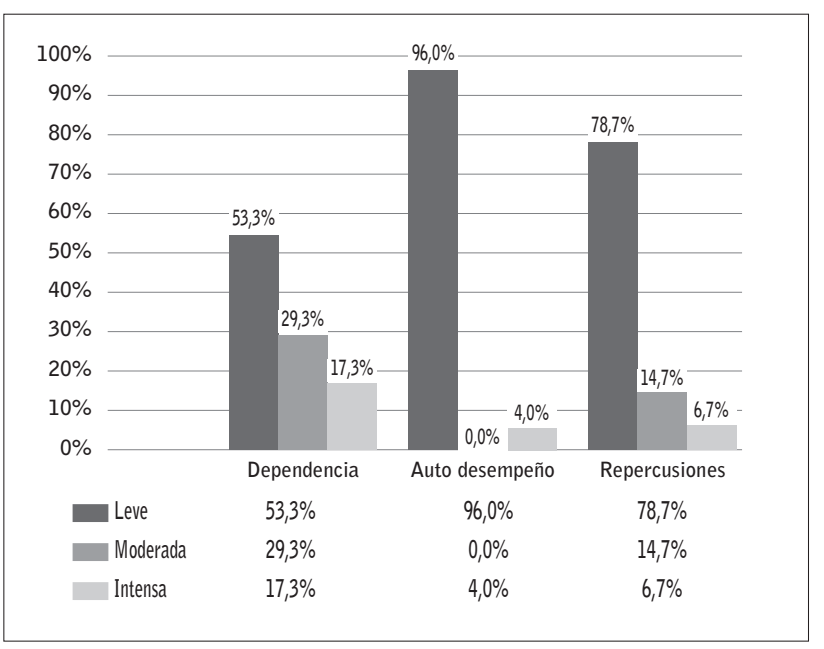

Figura 2. Dimensiones de sobrecarga del cuidador.

la determinación del nivel de sobrecarga del cuidador del paciente con tratamiento en DP. Estos datos son similares a la investigación referida a sobrecarga de los familiares cuidadores de pacientes con esquizofrenia ${ }^{5,6,7}$ que corrobora el nivel de sobrecarga en familiares cuidadores. Sin embargo, esta misma situación de sobrecarga del rol del cuidador se incrementa cuando el paciente se encuentra hospitalizado, según el estudio realizado en la ciudad de Mexico por Rivero García $R$, et al $^{8}$. La incertidumbre y el estrés de un ingreso hospitalario generó un aumento de la sobrecarga del rol del cuidador, encontrándose 126 ingresos y 24 reingresos, con estancia hospitalaria acumulada de 350 días, la escala de Zarit demostró sobrecarga en $60 \%$, de los cuales $67 \%$ presentaron sobrecarga intensa y $33 \%$ sobrecarga. Podemos destacar como el entorno familiar o el hogar donde se encuentra el cuidador principal, representa un medio donde los niveles de sobrecarga se disminuyen a diferencia de investigaciones donde el familiar cuidador se encuentra en la institución hospitalaria. Esta situación podría ser profundizada en futuras investigaciones para poder detallar la relación causal que percibimos con estas observaciones.

Las características sociodemográficas tienen relevancia en la sobrecarga del cuidador, determinan su perfil y su mayor probabilidad para asumir la responsabilidad de la atención de larga duración?. Estos datos Ilaman la atención porque a pesar de que el cuidador principal, tiene un trabajo fuera del hogar, son casados, son cuidadores por más de dos años, han mostrado un nivel de sobrecarga leve; esto explica nuevamente importancia de tener en cerca al entorno de hogar no siendo necesariamente representado por la estructura física sino por aquello subjetivo que existe pero no se ve y si se siente.
El apoyo que representa cada miembro de la familia para el mismo cuidador principal se pone en evidencia en la investigación realizada por Flores G. et al. ${ }^{9}$, la cual explica como el cuidador principal puede sobrellevar su carga con la organización del trabajo igualitaria y equitativa de los miembros de la familia; en ese estudio los cuidadores eran en su mayoría mujeres de 40 a 59 años, preuniversitarias, cónyuges del enfermo, sin vínculo laboral, convivían con este, lo cuidaban hacía más de 5 años a tiempo completo y lo atendían por razones afectivas ${ }^{10}$.

Es conocido que las personas que asumen el cuidado de una persona con problemas de dependencia suelen experimentar graves problemas de salud física y mental, así como consecuencias negativas sobre su bienestar psicológico derivados del estrés originado por las circunstancias en que se desarrolla el cuidado ${ }^{7}$. Situación diferente a la encontrada en la muestra de la investigación, donde según la percepción del cuidador en DP la tiene un nivel leve de sobrecarga. Este hallazgo es diferente a la investigación denominada: El cansancio del rol del cuidador que realizan tratamiento de hemodiálisis, empleó el Índice de Barthel, dando como resultado que la gran mayoría de los pacientes tienen una dependencia severa a moderada ${ }^{11}$; en el estudio denominado características de pacientes de enfermedades de Parkinson asociados con una mayor carga entre sus cuidadores, concluye que las alteraciones motoras, deterioro cognitivo y lo más importante es la depresión de los pacientes con enfermedad de Parkinson son determinantes de carga para sus cuidadores ${ }^{12}$.

Los cuidadores que se enfrentan por un periodo de tiempo a elevadas demandas de cuidado están expuestos a sobrecarga que repercute negativamente sobre su salud física y mental ${ }^{7}$. Respecto al auto desempeño del cuidador de los pacientes en DP son parecidos a los publicados en otros estudios, el cuidador informal primario, es aquel que proporciona la mayor parte de la asistencia y apoyo diario al enfermo, quienes utilizaron estrategias de afrontamiento centrado en el problema y en las emociones, sin embargo el $100 \%$ tuvo niveles medios y altos de ansiedad y depresión $n^{7,10}$.

Durante el cuidado a los pacientes que reciben DP, los cuidadores realizan múltiples tareas que pueden generar demandas físicas, emocionales, sociales o financieras, lo que repercutirá en las actividades de la vida cotidiana del cuidador (social, laboral y familiar). Al realizar el análisis de las repercusiones socio familiares del cuidador en DP presentó nivel leve con un 
$78,7 \%$, la cual coincide con el estudio de Velázquez $Y$, et al. Como afectación socioeconómica predominó la limitación del tiempo libre, poco apoyo percibido, escasas relaciones sociales, problemas económicos y dificultades laborales ${ }^{10}$.

La carga resultante del cuidado puede generar carga en varios dominios de la vida del cuidador: físico, psicológico, social, espiritual, y financieros. Sin embargo, el cuidador puede no percibir todas las dimensiones al mismo tiempo. Esta carga puede cambiar durante el tiempo a medida que el cuidador se adapta y encuentra nuevas maneras de hacer frente al deterioro del paciente, o se sustituyen por nuevos problemas 0 situaciones de crisis $^{13}$.

Este estudio tuvo como limitación la poca existencia de antecedentes con la variable independiente y particularmente de la unidad de análisis, esto último podría ser tomado en cuenta para destacar la relevancia y pertinencia de la investigación. Podemos concluir que, el nivel de sobrecarga del cuidador es leve, y el perfil más prevalente respecto a los datos sociodemográficos de los cuidadores es mujer adulta, esposa del paciente y que vive con el paciente.

\section{Agradecimientos}

A los cuidadores por su participación, y al personal de enfermería de la unidad de Diálisis Peritoneal por el apoyo al realizar la investigación.

Recibido: 12-02-19

Revisado: 05-03-19

Modificado: 13-04-19

Aceptado: 25-05-19

\section{Bibliografía}

1. Organización Panamericana de la Salud y Organización mundial de la salud. Estados unidos. [Consultado 03 agosto 2019]. Disponible en: http:// www.paho.org/hq/index.php?option=com_content\&view=article\&id=10542\%3A2015-opsoms-sociedad-latinoamericana-nefrologia-enfermedad-renal-mejorar-tratamiento\&Itemid=1926\&lang=es.

2. Sociedad Peruana de Nefrología. Perú. [Consultado 03 agosto 2019]. Disponible en: http://www.spn.pe/.

3. Hurtado I, Hurtado A, Cárceles E, Navarro J, Párraga $M$, Bolaños S. Sobrecarga de los cuidadores en diálisis peritoneal. Enferm Nefrol [Internet] 2015 [Consultado agosto 2019]; 18(1): [24-31 p.]. Disponible en: http://scielo.isciii.es/scielo.php?scrip$\mathrm{t}=$ sci_arttext\&pid=S2254-28842015000500020.

4. Teixidó J, Tarrats L, Arias N, Cosculluela A. Cuestionario de sobrecarga de cuidadores de pacientes de diálisis peritoneal. Rev Nefrología [Internet] 2006 Febrero [Consultado 03 agosto 2019]; 26 (1): [110 p.]. Disponible en: https://www.revistanefrologia.com/es-pdf-X0211699506019224.

5. Correa L, Diccia E. Nivel de sobrecarga de los familiares cuidadores de pacientes con esquizofrenia que asisten a un servicio de salud - 2014 [tesis]. Lima: Universidad Nacional Mayor de San Marcos [Internet] 2015 [Consultado 04 agosto 2019]; [aprox. 93 p.]. Disponible en: http://cybertesis.unmsm.edu.pe/ handle/cybertesis/4177.

6. Li C. Nivel de sobrecarga del cuidador del paciente con esquizofrenia y factores asociados en el Hospital Regional Docente Las Mercedes durante el periodo octubre-diciembre 2013 [Tesis]. Chiclayo: Universidad Católica Santo Toribio de Mogrovejo [Internet] 2014 [Consultado 04 agosto 2019]; [aprox. 32 p.]. Disponible en: http://hdl.handle. net/20.500.12423/303.

7. Li M, Alipázaga P, Osada J, León F. Nivel de sobrecarga emocional en familiares cuidadores de personas con esquizofrenia en un hospital público de Lambayeque - Perú. Rev Neuropsiquiatr [Internet] 2015 Octubre [Consultado 04 agosto 2019]; 78 (4): [1-8 p.]. Disponible en: http://www. scielo.org.pe/scielo.php?script=sci_arttext\&pi$d=$ S0034-85972015000400006\&lng =es\&nrm $=i-$ so\&tlng=es.

8. Rivero R, Segura E, Juárez N. Overload of primary caregivers for patients on hemodialysis generated by hospital readmissions. Diálisis y Trasplante [Internet] 2015 Julio-Diciembre [Consultado 03 agosto 2019]; 36(2): [72-77 p.]. Disponible en: https:// doi.org/10.1016/j.dialis.2015.02.001. 
9. Flores E, Rivas E, Seguel F. Nivel de Sobrecarga en el Desempeño del Rol del Cuidador Familiar de Adulto Mayor con Dependencia Severa. Cienc. Enferm [Internet] 2012 Abril [Consultado 05 agosto 2019]; 18 (1): [29-41 p.]. Disponible en: http:// dx.doi.org/10.4067/S0717-95532012000100004.

10. Velázquez Y, Espín AM, Repercusión psicosocial y carga en el cuidador informal de personas con insuficiencia renal crónica terminal. Rev Cubana Salud Pública [Internet] 2014 [Consultado 05 agosto 2019]; 40(1): [1-16 p.]. Disponible en: http://www. redalyc.org/articulo.oa?id=21430496002.

11. Gil I, Pilares JA, Romero R, Santos M. Cansancio del rol de cuidador principal del paciente en hemodiálisis. Enferm Nefrol [Internet] 2013 [Consultado 06 agosto 2019]; 16 (1): [156-217 p.]. Disponible en: http://dx.doi.org/10.4321/S225428842013000500087.

12. Benavides 0 , Alburquerque D, Chaná P. Evaluación de la sobrecarga en los cuidadores de los pacientes con enfermedad de Parkinson ambulatorios y sus factores de riesgo. Rev Méd Chile [Internet] 2013 marzo [Consultado 06 agosto 2019]; 141(3): [320-6 p.]. Disponible en: http://dx.doi.org/10.4067/ S0034-98872013000300006.

13. Kuei-Ru Chou, PhD, RN. Caregiver Burden: A Concept Analysis. Journal of Pediatric Nursing. [Internet] 2000 December [Consultado 04 agosto 2019]; 15 (6). Disponible en: https://doi.org/10.1053/ jpdn.2000.16709.

Este artículo se distribuye bajo una Licencia Creative Commons Atribución-NoComercial 4.0 Internacional. https://creativecommons.org/licenses/by-nc/4.0/

Open Access (C) (;) (8) 